\title{
Design and Uncertainty Evaluation of the Tiny Gas Flow Calibrator
}

\author{
CHEN Pan-pan ${ }^{1,2}$, CHENG Jia ${ }^{2,3}$, WANG Wen-lin ${ }^{2}$, SHEN Wen-xin ${ }^{2}$, XIE \\ Dai-liang ${ }^{1}$ \\ ${ }^{1}$ China Jiliang University, Hangzhou 310018,China \\ ${ }^{2}$ Zhejiang Institute of Metrology, Hangzhou 310018, China \\ ${ }^{3}$ Zhejiang intellectual property service center, Hangzhou 310012, China
}

\begin{abstract}
Keywords: tiny flow;Symmetric plunger; calibrator; uncertainty
\end{abstract}
\begin{abstract}
In recent years, the measurement and controlment of the tiny gas flow have been gained widely attention in some emerging areas, such as biological, medical, aerospace and so on. In order to study the characteristics of the tiny gas flow meter, the tiny gas flow calibrator based on the principle of the Symmetric plunger is designed, which the flow range of the calibrator is from $5 \mathrm{~mL} / \mathrm{h}$ to $1000 \mathrm{~mL} / \mathrm{h}$. The structure and the working principle are introduced, the uncertainty's Sources and classification of the tiny gas flow calibrator are analyzed, the uncertainty components are analyzed and calculated, The expanded uncertainty of the device is less than $0.126 \%$, which conforms to the design requirements of the device, and it have reached the advanced level at home and abroad. Finally, according to the experiment of the soap film flowmeter, The expanded uncertainty of calibration for the soap film flowmeter is less than $0.77 \%$.
\end{abstract}

\section{Introduction}

With the rapid development and application of the technology, some emerging research areas have been gained widely attention in recent years, such as biological, medical, aerospace, and so on. The flow measurement have been extend in the direction of small flow rate, so the measurement and controlment of the tiny gas flow have been gained widely attention in those emerging areas, the calibration for the tiny gas flow meter has become one of the important research content in the current flow metrology areas[5,7,8].

Some tiny gas flow standard devices have been established in many famous measurement organizations at home and abroad, such as the country's supreme tiny gas flow calibration device in NIST of the USA, which the flow range of the calibrator is from $0.03 \mathrm{~L} / \mathrm{min}$ to $30 \mathrm{~mL} / \mathrm{min}$, the uncertainty of the calibrator is $0.2 \%(k=2)[9]$; the piston calibrator in PTB of the Germany, which the flow range of the calibrator is from $5 \mathrm{~mL} / \mathrm{h}$ to $5 \mathrm{~L} / \mathrm{h}$, the uncertainty of the calibrator is $0.05 \%(k=2)$ [2]; the Soap membrane type gas flow standard device in the research of metrology of China, which the flow range of the calibrator is from $25 \mathrm{~mL} / \mathrm{min}$ to $500 \mathrm{~mL} / \mathrm{min}$, the uncertainty of the calibrator is $1.0 \%(k=2)$. In comparison, China's domestic starts later than the foreign in terms of small flow measurement, the study is also relatively small, the gap with foreign is very obvious[3,10].

In order to adapt to the requirements of more and more tiny gas flow measurements, In this paper, on the basis of previous studies[1,4,6], the tiny gas flow calibrator based on the principle of the Symmetric plunger is designed, which the flow range of the calibrator is from $5 \mathrm{~mL} / \mathrm{h}$ to $1000 \mathrm{~mL} / \mathrm{h}$, the effective volume is $44 \mathrm{~mL}$, the uncertainty of the calibrator is $(0.1-0.3) \%(k=2)$.

\section{Standard device}

The way of the electromechanical combination is used in this tiny gas flow calibrator(Hereinafter referred to as "calibrator"), ball screw is driven by a motor, and then drive the symmetric plunger to move forward, in a certain time, the flow meter is calibrated by the standard volume, which produces by the piston movement displacement.

Fig. 1 is the principle diagram of the standard device, its Structure is mainly composed of the 
following sections: Air supply part, mainly composed by the high pressure gas source, drying device, two levels of pressure regulating mechanism and buffer tank and so on, among them, different temperament of the compressed gas for the experiment can be choosed as the gas source, through regulating mechanism, the detected flowmeter can be calibrated under the rated pressure; Symmetric plunger parts, mainly composed by the structure of the Symmetric plunger and its affiliated branches, among them, a big traffic flow indicator F1 and two-way small flow indicator F2 are mounted on the branches, the chambers which before and after the branch are connected to each other, and the adjustment of the flow controller to complete the flow point for the calibration; Control, data acquisition and processing parts, mainly composed by the hardware and software of the processor system, and upper software, the plant data acquisition, motion control, process control and data storage are completed, and so on.

The Symmetric plunger is used as the volume measurement standard in the calibrator, the chambers around the Symmetric plunger are connected to each other, the pressure of the chambers is equal basically, in the process of the calibration, the gas can not be extrusion or pull away with the movement of the plunger, and the Motor's driven load does not increase, because the plunger volume accuracy is higher, in the entire calibration process, the flow rate and the stability of state parameter can be better maintained, so it has great advantage in tiny gas flow measurement.

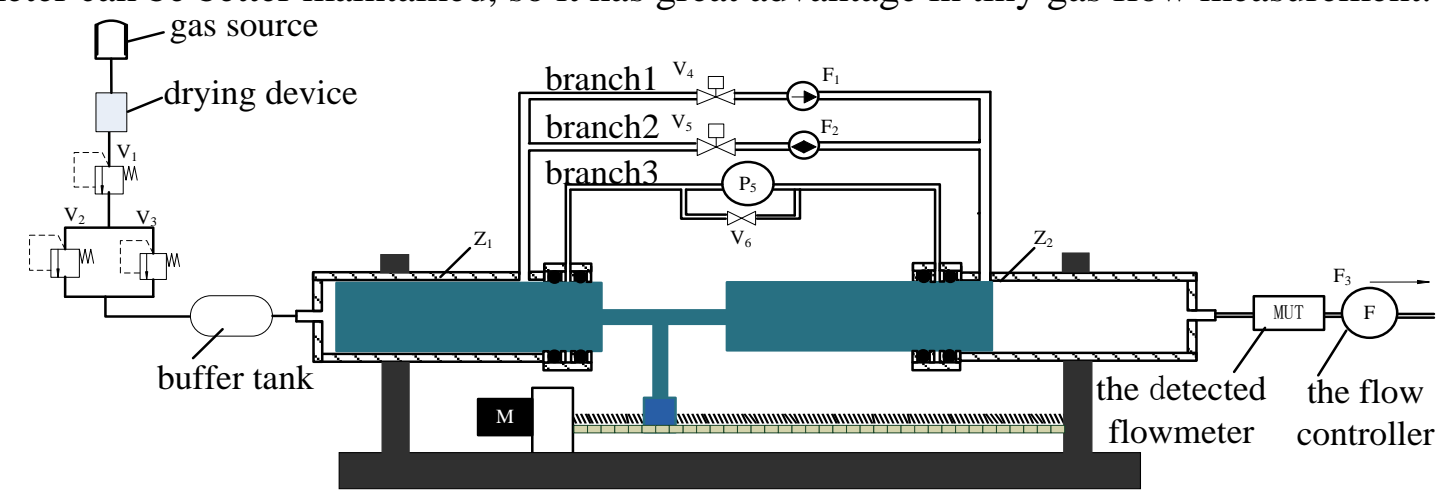

Fig.1 The schematic diagram of the tiny gas flow calibrator

The positive pressure is used in the calibration, specific implementation as follows: before the calibration, the gas outlet pressure is dispatched to checked to the rated working pressure of the detected flowmeter after two level of pressure regulating, the gas flow is adjusted to the calibration points by the flow indicator, then open the valve 4, close the valve 5 , the high pressure gas is transfered from the chamber Z1 to the chamber Z2 though the valve 4 and the flow indicator F1 in the branch 1 , and then through the detected flowmeter. At the start of the calibration, the motor is drived according to the calibration's flow values of the detected flowmeter, the plunger is moved into the chamber Z2,and pull away from the chamber Z1 at the same time, the space made by the extrusion of the Symmetric plunger is filled by the upstream gas, in this process, the value of the flow indicator F1 decreases.After the value of the flow indicator F1 stables, open the valve 5, close the valve 4, the high pressure gas is transfered from the branch 1 to the branch 2, and record the value of F2 as the compensation flow when it stables, the standard flow of the detected flowmeter is calculated according to the pressure and temperature of the gas in the chambers, the time and the effectively movement distance of the plunger. the sum of the compensation flow and the standard flow which made by the symmetric plunger is used as the standard flow, the detected flowmeter is calibrated by the standard flow.

\section{Uncertainty analysis}

In the process of the calibration, the motor is controlled by the computer, driving the plunger translational motion forward, according to formula(1), the value of the gas's mass flow $Q_{s}$ made by the movement of the symmetric plunger is calculated. 
$Q_{s}=\rho \cdot Q_{v}=\rho \cdot \frac{\pi \cdot d_{k}^{2} \cdot L}{4 t}$

Here, $\rho$ is the gas density in working condition, $\mathrm{kg} / \mathrm{m}^{3} ; Q_{\mathrm{v}}$ is the volume flow made by the movement of the symmetric plunger, $\mathrm{m}^{3} / \mathrm{h} ; d_{k}$ is the diameter of the plunger, $\mathrm{mm}$; $L$ is the movement distance of the plunger,mm; $t$ is the movement time of the plunger,s.

According to formula(2), the value of the gas density in working condition is calculated.

$\rho=\rho_{0} \cdot \frac{P \cdot T_{0}}{T \cdot P_{0}}$

Here, $P$ is the pressure in working condition, $P$ a; $P_{0}$ is the Standard atmospheric pressure, $101325 \mathrm{~Pa} ; \mathrm{T}$ is the temperature in working condition, $\mathrm{K} ; T_{0}$ is the gas temperature in standard condition, $273.15 \mathrm{~K} ; \rho_{0}$ is the atmospheric density in standard condition, $1.293 \mathrm{~kg} / \mathrm{m}^{3}$. According to formula(3), the value of the gas's mass flow $Q_{\mathrm{s}}$ made by the movement of the symmetric plunger is calculated.

$$
Q_{s}=\rho_{0} \cdot \frac{P \cdot T_{0}}{T \cdot P_{0}} \cdot \frac{\pi \cdot d_{k}^{2} \cdot L}{4 t}
$$

According to formula (3), the uncertainty's sources of the calibrator can be considered from the following several aspects:

(1) the uncertainty introduced by the diameter $D$ of the plunger;

(2) the uncertainty introduced by the movement distance $L$ of the plunger;

(3) the uncertainty introduced by the pressure $P$ in the chambers;

(4) the uncertainty introduced by the temperature $T$ in the chambers;

(5) the uncertainty introduced by the movement time $t$ of theplunger.

$u_{a}(D)$ which introduced by the diameter $D$ of the plunger mainly composed by the following parts: the uncertainty $u_{a 1}\left(D_{1}\right)$ introduced by the diameter $d_{k}$ of the plunger and the uncertainty $u_{a 2}\left(D_{2}\right)$ introduced by the improper installation.

The diameter $D$ of the plunger is $16 \mathrm{~mm}$, the Maximum cylindricity error of the plunger's diameter is $0.01 \mathrm{~mm}$, according to the rectangular distribution, $k=\sqrt{3}$, according to formula(4), the Class $B$ relatively standard uncertainty of measurement is calculated.

$$
u_{a 1}\left(D_{1}\right)=\frac{0.01}{16 \times \sqrt{3}}=0.036 \%
$$

The maximum allowable slope angle on the horizontal direction of the plunger in the process of the installation is $\pm 0.5^{\circ}$, the maximum movement distance of the plunger is $20 \mathrm{~mm}$, according to formula(5), the maximum error introduced by the installation tilt of the plunger is calculated.

$$
u_{a 2}\left(D_{2}\right)=\frac{20-20 \times \cos 0.5^{\circ}}{20}=0.0038 \%
$$

According to formula (6), the total measurement uncertainty introduced by the diameter $D$ of the plunger is calculated.

$$
u_{a}(D)=\sqrt{u_{a 1}^{2}\left(D_{1}\right)+u_{a 2}^{2}\left(D_{2}\right)}=0.036 \%
$$

$u_{b}(L)$ which introduced by the movement distance $L$ of the plunger mainly composed by the following parts: the uncertainty $u_{b 1}\left(L_{1}\right)$ introduced by the motor and the uncertainty $u_{b 2}\left(L_{2}\right)$ introduced by the screw.

The model of the motor is VRDM364, 200 step in one turn, the maximum System angle error of the motor is 6', the minimum measurement time in $5 \mathrm{ml} / \mathrm{min}$ is $1200 \mathrm{~s}$, the minimum movement distance of the screw is $30 \mathrm{~mm}$, the motor has rotated 15 , according to formula(7), the uncertainty introduced by the motor is calculated.

$$
u_{b 1}\left(L_{1}\right)=\frac{6}{200 \times 15 \times 1.8 \times 60}=0.002 \%
$$

The model of the screw is the BNK series of the THK company, the cumulative lead error of the screw is $\pm 0.008 \mathrm{~mm} / 300 \mathrm{~mm}$, according to the rectangular distribution, $k=\sqrt{3}$, according to formula(8), the Class $B$ relatively standard uncertainty of measurement is calculated. 


$$
u_{b 2}\left(L_{2}\right)=\frac{0.008}{30 \times \sqrt{3}}=0.015 \%
$$

According to formula(9), the total measurement uncertainty introduced by the movement distance $L$ of the plunger is calculated.

$$
u_{b}(L)=\sqrt{u_{b 1}^{2}+u_{b 2}^{2}}=0.015 \%
$$

$u_{c}(P)$ which introduced by the pressure $P$ in the chambers mainly composed by the following parts: the error value $u_{c 1}\left(P_{1}\right)$ introduced by the Gage pressure sensor, the error value $u_{c 2}\left(P_{2}\right)$ introduced by the absolute pressure sensor, and the error value $u_{c 3}\left(P_{3}\right)$ introduced by the Cylinder pressure fluctuations.

The absolute pressure of the flow is $201325 \mathrm{~Pa}$, the measuring range of the Gage pressure sensor is $400 \mathrm{kPa}$, the precision is $0.04 \%$, the measuring range of the absolute pressure sensor is $120 \mathrm{kPa}$, the precision is $0.04 \%$, according to the rectangular distribution, $k=\sqrt{3}$, according to formula(10-11), the Class $B$ relatively standard uncertainty of measurement is calculated.

$$
\begin{aligned}
& u_{c 1}\left(P_{1}\right)=\frac{4 \times 10^{-4}}{\sqrt{3}}=0.023 \% \\
& u_{c 2}\left(P_{2}\right)=\frac{4 \times 10^{-4}}{\sqrt{3}}=0.023 \%
\end{aligned}
$$

The maximum pressure fluctuations in the chambers in the process of the calibration though the early simulation research is $10 \mathrm{~Pa}$, according to the rectangular distribution, $k=\sqrt{3}$, according to formula(12), the Class $B$ relatively standard uncertainty of measurement is calculated.

$$
u_{c 3}\left(P_{3}\right)=\frac{10}{201325 \times \sqrt{3}}=0.0028 \%
$$

According to formula(13), the total measurement uncertainty introduced by the pressure $P$ in the chambers is calculated.

$$
u_{c}(P)=\sqrt{u_{c 1}^{2}\left(P_{1}\right)+u_{c 2}^{2}\left(P_{2}\right)+u_{c 3}^{2}\left(P_{3}\right)}=0.033 \%
$$

$u_{d}(T)$ which introduced by the temperature $T$ in the chambers mainly composed by the following parts:the error value $u_{d 1}\left(T_{1}\right)$ introduced by the temperature sensor, and the error value $u_{d 2}\left(T_{2}\right)$ introduced by the temperature fluctuations.

The temperature of the flow is $293 \mathrm{~K}$, the precision is $0.15 \mathrm{C}$, according to the rectangular distribution, $k=\sqrt{3}$, according to formula(14), the Class $B$ relatively standard uncertainty of measurement is calculated.

$$
u_{d 1}\left(T_{1}\right)=\frac{0.15}{293 \times \sqrt{3}}=0.03 \%
$$

The maximum temperature fluctuations in the chambers in the process of the calibration though the early simulation research is $10 \mathrm{~Pa}$, according to the rectangular distribution, $k=\sqrt{3}$, according to formula(15), the Class $B$ relatively standard uncertainty of measurement is calculated.

$$
u_{d 2}\left(T_{2}\right)=\frac{0.1}{293 \times \sqrt{3}}=0.02 \%
$$

According to formula(16), the total uncertainty introduced by the temperature $T$ in the chambers is calculated.

$$
u_{d}(T)=\sqrt{u_{d 1}^{2}\left(T_{1}\right)+u_{d 2}^{2}\left(T_{2}\right)}=0.036 \%
$$

The allowed error of the time measuring with experiment interface card is \pm 100 clock cycles, the fundamental frequency of the clock is $1 \mathrm{MHz}$, according to the rectangular distribution, $k=\sqrt{3}$, according to formula(17), the Class $B$ relatively standard uncertainty of measurement is calculated.

$$
u_{e}(t)=\frac{100}{10^{6} \times \sqrt{3}}=0.006 \%
$$

According to formula(18), the synthetic standard uncertainty of the calibrator is calculated.

$$
u_{Q}=\sqrt{u_{a}^{2}(D)+u_{b}^{2}(L)+u_{c}^{2}(P)+u_{d}^{2}(T)+u_{e}^{2}(t)}=0.063 \%
$$


According to formula(19), the expanded uncertainty of the calibrator with $k=2$ is calculated.

$U(Q)=k \cdot u_{Q}=2 \times 0.054 \%=0.126 \%$

\section{Calibration experiment}

In order to inspect the ability of the calibrator, certain numbers of calibration experiments for the flowmeter with the the calibrator are made, the smart soap film flowmeter which the flow range is from $2 \mathrm{~mL} / \mathrm{min}$ to $20 \mathrm{~mL} / \mathrm{min}$, and the model is HY-5020 is used as the detected flowmeter, the resolution of the smart soap film flowmeter is $0.1 \mathrm{~mL} / \mathrm{min}$. The experiments are took under the condition of the room temperature 20C, the indoor relative humidity $60 \%$, select some flow points, such as 2, 6, 10, 15, and 19 and so on, every flow point measures four times, the experiment results are shown in the table 1.

Tab.1 The experimental data of the HY-5020 Smart soap film flowmeter

\begin{tabular}{ccccccc}
\hline $\begin{array}{c}\text { Flow points } \\
(\mathbf{m L} / \mathbf{m i n})\end{array}$ & $\begin{array}{c}\text { Bypass flow } \\
(\mathbf{m L} / \mathbf{m i n})\end{array}$ & $\begin{array}{c}\text { Actual flow } \\
(\mathbf{m L} / \mathbf{m i n})\end{array}$ & $\begin{array}{c}\text { Meter reading } \\
(\mathbf{m L} / \mathbf{m i n})\end{array}$ & $\begin{array}{c}\overline{\mathbf{Q}}_{\mathrm{m}} \\
(\mathbf{m L} / \mathbf{m i n})\end{array}$ & $\begin{array}{c}\text { error } \\
(\mathbf{\%})\end{array}$ & $\begin{array}{c}\text { Repetitive } \\
(\mathbf{\%})\end{array}$ \\
\hline \multirow{3}{*}{19} & 0.0281 & 19.0281 & 19.10 & & & \\
& 0.0314 & 19.0314 & 19.03 & 19.04 & 0.21 & 0.33 \\
& 0.0316 & 19.0316 & 19.06 & & & \\
& 0.0305 & 19.0305 & 18.95 & & & \\
15 & 0.0256 & 15.0256 & 15.05 & & & \\
& 0.0164 & 15.0164 & 15.03 & 15.02 & 0.13 & 0.26 \\
& 0.0242 & 15.0242 & 14.96 & & & \\
& 0.0246 & 15.0246 & 15.02 & & & \\
10 & 0.0221 & 10.0221 & 10.03 & & & \\
& 0.0168 & 10.0168 & 9.89 & 9.98 & & \\
& 0.0164 & 10.0164 & 10.05 & & & \\
& 0.0174 & 10.0174 & 9.94 & & & \\
6 & 0.0156 & 6.0156 & 6.06 & & & \\
& 0.0156 & 6.0156 & 6.01 & 6.03 & 0.5 & \\
& 0.0141 & 6.0141 & 6.00 & & & \\
& 0.0134 & 6.0134 & 6.03 & & & \\
& 0.0055 & 2.0055 & 2.01 & & & \\
\hline
\end{tabular}

The uncertainty of the HY-5020 smart soap film flowmeter can be considered from the following several aspects:

(1) the uncertainty introduced by the experimental data repeatability;

(2) the uncertainty introduced by the bypass flow indicator;

(3) the uncertainty introduced by the device.

According to the table 1 , the maximum repetitive error which appeared in the flow of $10 \mathrm{~mL} / \mathrm{min}$ is $0.76 \%$, according to formula(20), the uncertainty introduced by the experimental data repeatability is calculated.

$$
u_{1}=\frac{E}{\sqrt{n}}=0.38 \%
$$

The precision grade of the bypass flow indicator is 2 , according to the table 1 , the maximum bypass flow error is appeared in the $2 \mathrm{~mL} / \mathrm{min}$, according to formula(21), the uncertainty introduced by the bypass flow indicator is calculated.

$$
u_{2}=\frac{0.0073 \times 2 \times 10^{-2}}{2}=0.0073 \%
$$

The standard uncertainty introduced by the device is $0.063 \%$,according to the uncertainty 
analysis before. according to formula(22), the synthetic standard uncertainty of the HY-5020 smart soap film flowmeter is calculated.

$$
u=\sqrt{u_{1}^{2}+u_{2}^{2}+u_{Q}^{2}}=0.385 \%
$$

According to formula(23), the expanded uncertainty of the HY-5020 smart soap film flowmeter with $k=2$ is calculated.

$U=k \cdot u=0.77 \%$

\section{Conclusions}

The working principle of the tiny gas flow calibrator is introduced, the uncertainty's sources of the calibrator are analyzed, the expand uncertainty of the calibrator with $k=2$ is $0.126 \%$, it conforms to the design requirements of the calibrator, ruduces the measurement error in the process of the dissemination, and improves the degree of the reliable measurement results. Finally, the calibrator is used to the calibration experiments for the HY-5020 smart soap film flowmeter, the synthetic standard uncertainty of the HY-5020 smart soap film flowmeter is $0.77 \%$. At the same time, the calibrator can also be test for other small gas flow meter calibration, such as mass flowmeter, flow meter, the subsequent calibration experiments are proceed in the further.

\section{References}

[1] Chang Xiangyue, Cheng Jia, Wang Wenlin, et al. 2013. Design and simulation of the symmetric plunger tiny gas prover[J].the academic journal of the china jiliang university, 24(4):347-35.

[2] Kramer R B, Mickan B, Dopheide D. 2006. Double piston prover usable as flow-rate comparator for various gases[A]. In: XVIII IMEKO WORLD CONGRESS[C]. Rio de Janeiro: International Measurement Confederation, CD-ROM, paper 1, 2 Seiten.

[3] Shen Wenxin, Jin Lan, Chen Shangshun. 2008. Research on the piston of the gas flow calibrator based on the principle of the Balance adjustment[J]. China metrology, (9):58-59.

[4] Shen Wenxin, Zhan Zhijie, Jin Lan, et al. 2011. Research on the Critical flow nozzle dynamic test method based on the principle of the conservation of mass[J].Journal of measurement, 32(3):213-216.

[5] Su Junmin, Cai Kunzhi, Yang Zhengcai, et al. 2008. Biomedical chip with micro meter technology[J]. Measurement technology, (9):129-137.

[6] Teng Penghui, Cheng Jia, Yang Qihua. 2012. Research of piston pump analyzer test equipment[J].Sensor and micro system,31(12):16-18.

[7] Wang Guina, Su Xiaoguang. 2007. Research on the tiny gas flow detection technology[J]. Test and technology of China, 32(6): 52-54.

[8] Wu S, Lin Q, Yuen Y, et al. 2001. MEMS flow sensors for nano-fluidic applications[J]. Sensors and Actuators A: Physical, 89(1): 152-158.

[9] Yeh T T, Aguilera J, John D W. 2005. Hydrocarbon liquid flow calibration service[M]. Gaithersburg: NIST.

[10] Zhang Yongsheng, Bai Yin. 2011. The gas flow calibrator based on the principle of the Passive piston[J]. Space measuring technology, 31(1):67 70. 